\title{
Predicting Traffic Congestion Evolution: A Deep Meta Learning Approach
}

\author{
Yidan Sun, Guiyuan Jiang*, Siew-Kei Lam and Peilan He \\ Nanyang Technological University, Singapore \\ ysun014@e.ntu.edu.sg, \{gyjiang, assklam\}@ntu.edu.sg, phe002@e.ntu.edu.sg.
}

\begin{abstract}
Many efforts are devoted to predicting congestion evolution using propagation patterns that are mined from historical traffic data. However, the prediction quality is limited to the intrinsic properties that are present in the mined patterns. In addition, these mined patterns frequently fail to sufficiently capture many realistic characteristics of true congestion evolution. In this paper, we propose a representation learning framework to characterize and predict congestion evolution between any pair of road segments. Specifically, we build dynamic attributed networks (DAN) to incorporate both dynamic and static impact factors while preserving dynamic topological structures. We propose a Deep Meta Learning Model (DMLM) for learning representations of road segments which support accurate prediction of congestion evolution. DMLM relies on matrix factorization techniques and meta-LSTM modules to exploit temporal correlations at multiple scales, and employ meta-Attention modules to merge heterogeneous features while learning the time-varying impacts of both dynamic and static features. Compared to all state-of-the-art methods, our framework achieves significantly better prediction performance on two congestion evolution behaviors (propagation and decay) when evaluated using real-world dataset.
\end{abstract}

\section{Introduction}

Accurate prediction of congestion evolution (such as how the congestion propagates or diminishes across road segments) benefits many traffic management applications, such as traffic prediction [Nguyen et al., 2016], route planning, and bottleneck identification. Existing works on congestion evolution prediction typically rely on data mining techniques [Nguyen et al., 2016]. In these works, pattern mining is applied on historical traffic data to obtain frequent patterns from causality tree/graph [An et al., 2016; An et al., 2018]. These patterns (subtrees, subgraphs) are then utilized for congestion evolution prediction [Nguyen et al., 2016;

\footnotetext{
* corresponding author
}

Xiong et al., 2018]. The quality of such predictions is limited to the inherent properties found in the available patterns. These evolution patterns usually cannot sufficiently capture many realistic characteristics of true congestion evolution such as the significant variation in spatiotemporal range and granularity, and the asymmetric transitivity of congestion propagations.

Factors that will impact congestion evolutions include road characteristics (e.g., road type), Points of Interests (POIs, e.g, school, shopping mall) [Zhang et al., 2017], and weather conditions. However, existing works often do not consider the extent of influence that these factors have on congestion evolution, such as the time-varying impact of static factors (e.g., POIs) [Liao et al., 2018]. For example, a stadium holding large social event (e.g. football game) is likely to cause unexpected congestions; schools tend to affect the traffic only during morning peak, lunch time, and evening peak hours. In this paper, we develop methods to incorporate the abovementioned factors with time-varying impact on congestions to predict the congestion evolution between any road pairs.

The contributions of our work are summarized as follows.

- We propose a representation learning framework to characterize and predict congestion propagation/decay between any pair of road segments. This departs from existing methods that mostly rely on causality trees/graphs and mining propagation patterns.

- We are the first to characterize traffic congestion evolution (propagation/decay) using dynamic attributed networks (DANs). Various factors with time-varying impacts are incorporated into DANs as node attributes. These include static attributes (e.g., POIs, road characteristics) and dynamic attributes (e.g., traffic speed).

- We propose a Deep Meta Learning Model (DMLM) for learning representations of road segments that support accurate prediction of congestion evolution. Meta learning is used to learn the evolving strengths of temporal correlations at multiple scales based on DAN attributes. DMLM also employs meta-Attention modules to merge heterogeneous features while learning the time-varying impacts of both dynamic and static features.

- The performance of our framework on two congestion evolution behaviors, congestion propagation and congestion decay, is evaluated using real-world dataset. Re- 
sults show that our framework significantly outperforms all state-of-the-art methods.

\section{Related Work}

Traffic Congestion Evolution. Many efforts have been undertaken to model congestion evolutions at three levels of granularity, i.e., macroscopic, microscopic, and mesoscopic. Macroscopic level formulates congestion as a cluster of likewise congested roads [Anwar et al., 2016; An et al., 2016], while microscopic level studies congestion evolution among subsections within an individual road [Sun et al., 2018; Khajeh-Hosseini and Talebpour, 2019; Cui et al., 2019]. Our work focuses on mesoscopic level, which associates congestion with individual road segment and congestion evolution across adjacent road segments. [Nguyen et al., 2016; Di et al., 2019; Yue et al., 2018] formulate congestion propagations as directed edges from downstream to upstream roads. Causality trees and graphs are constructed based on the edges and data mining techniques are used to obtain frequent subtrees/subgraphs as evolution patterns (e.g., decay and propagation). Some works also considered congestion evolution prediction based on the obtained evolution patterns. Most works at mesoscopic level rely on tree or graph structure to mine patterns and make predictions. However, such representation limits the design space of predictors due to the dependency on structural patterns.

Link Prediction on Network. The core idea of congestion propagation prediction is similar to link prediction on complex networks (e.g., social networks), which predicts new relationships (links). Similarity based methods, which assumes that two nodes are similar if they in close proximity or are connected to similar nodes, are most widely used for link prediction [Martínez et al., 2016b]. Local similarity is learned based on local neighborhood information [Martínez et al., 2016a], while global similarity relies on the topological information of the entire network [Lichtenwalter et al., 2010]. In general, similarity-based methods only focus on static structural information and neglect the evolving changes in the networks. In practice, nodes are often affiliated with rich attributes that co-evolve with network structures. As such, there has been a lot of interest to incorporate structure information and node attributes for link prediction [Li et al., 2018; Zhang et al., 2018]. However, existing works typically ignore the temporal characteristics in dynamic networks, and fail to capture long and short term node interactions. Link prediction on DANs is still currently an emerging area of research. In this paper, we study evolution prediction that takes into account dynamic traffic congestion networks (evolving topological structures), and their temporal characteristics (timevarying impacts of various factors on congestion evolution).

\section{Constructing Congestion Evolution Networks}

This section presents our method to construct attributed networks for modeling congestion evolution (propagation and decay), as shown in Figure 1 (a). The attributed networks capture dynamic congestion evolution using various types of

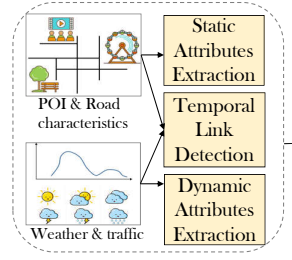

(a) Constructing $G_{p}$.

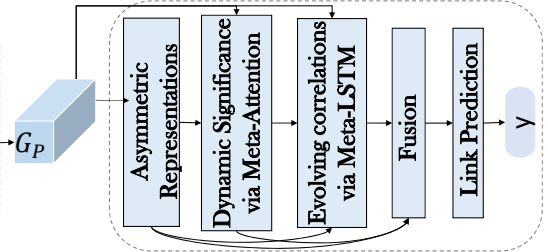

(b) DMLM.
Figure 1: Main framework: (a) Modeling congestion evolution using attributed propagation/decay networks. (b) Deep Meta Learning Model for congestion evolution prediction.

temporal links, while incorporating both static and dynamic node attributes.

Traffic Network. The traffic network is represented as a DAN $G_{C}=\left\{G_{C}^{t}\right\}$, where $G_{C}^{t}=\left\{V, E, \mathbf{C}^{t}\right\}, t$ is time slice ( $1 \leq t \leq T$, there are $T$ time slices in total), $V=$ $\left\{r_{1}, \ldots, r_{N}\right\}$ is a set of $N$ vertice (each vertex is a road segment), $E=\{(i, j)\}$ is a set of undirected edges (an edge $(i, j) \in E$ indicates that $r_{i}$ and $r_{j}$ are spatially connected). $V$ and $E$ capture static topology information that remains unchanged over time. Matrix $\mathbf{C}^{t} \in\{0,1\}^{N}$ represents traffic congestion of all $N$ road segments at time $t . c_{i}^{t}$ is an element in $\mathbf{C}$ where $c_{i}^{t}=1$ if road $r_{i}$ 's traffic state is congested at time $t$, otherwise, $c_{i}^{t}=0$. To capture congestion propagation and decay behaviors on the traffic network, two DANs, $G_{P}$ and $G_{D}$, are constructed based on $G_{C}$, as shown in Figure 2.

Attributed Propagation Network. $G_{P}^{t}=$ $\left(V_{P}^{t}, E_{P}^{t}, A D_{P}^{t}, A S_{P}^{t}\right)$, where set $V_{p}^{t} \subseteq V$ is a subset of $V$ which includes road segments involved in propagations; $E_{P}^{t}=\{(i, j)\}$ is a set of directed links, $(i, j) \in E_{P}^{t}$ represents a congestion propagation from $r_{i}$ to $r_{j}$ at time $t ; A D_{P}^{t}$ is a matrix of dynamic attributes associated with set $V_{P}^{t}$ and each vertex has $F_{d}$ dynamic attributes (e.g., traffic speed, time indicator, weather); $A S_{P}^{t}$ is a matrix of static attributes associated with set $V_{P}^{t}$, and each vertex has $F_{s}$ static attributes (e.g., POIs, road characteristics). The DAN $G_{P}$ is constructed in the following way, as shown in Figure. 2 (a). The congestion states at time $t$ and $t+1$ are illustrated in the upper box, based on which a set of congestion propagation paths are detected. Specifically, road $r_{5}$ is congested at time $t$, then road $r_{3}$ and $r_{1}$ become congested from time $t$ to time $t+1$. This indicates that the congestion on $r_{5}$ propagates to $r_{3}$ and $r_{1}$ (similarly, $r_{6}$ propagates its congestion to $\left.r_{7}\right)$. Formally, a path $p a=\left\langle r_{i_{1}}, . ., r_{i_{k}}, \ldots, r_{i_{K}}\right\rangle$ is a propagation path if $\left(i_{j}, i_{j+1}\right) \in E(1 \leq j \leq K-1)$; $c_{i_{1}}^{t}=1, c_{i_{j}}^{t}=0$ and $c_{i_{j}}^{t+1}=1(2 \leq j \leq \bar{K})$. All nodes in the obtained propagation paths are included in set $V_{P}^{t}$, and any link appearing in the propagation paths is included in set $E_{P}^{t}$. The static attributes $A S_{P}^{t}$ contain two parts: road characteristics and surrounding POIs. Road characteristics contain road length, number of lanes, number of bus stops and traffic signals, and road type, where one-hot encoding is applied to distinguish between 19 road types (e.g., highway, primary road, etc.). The POIs are grouped into 14 categories (Section 5.1). The POI distribution around a road segment 


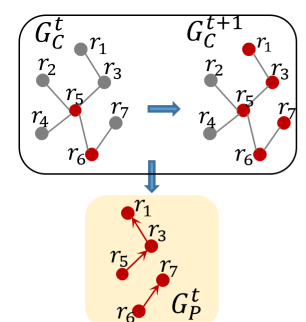

(a) Example for constructing $G_{P}^{t}$

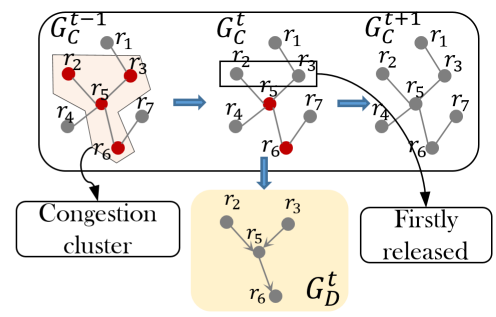

(b) Example for constructing $G_{D}^{t}$
Figure 2: Examples of constructing $G_{P}^{t}, G_{D}^{t}$ from $G_{C}$. Red and gray dots are congested and free-flow road segments, respectively.

$r_{i}$ (located in a circle area centered at the middle point of $r_{i}$ ) is formulated as a vector of the number of POIs in each category. Thus, each $r_{i}$ has a vector of dimension 14. The dynamic attributes $A S_{P}^{t}$ of $r_{i}$ at time $t$ contain: historical speeds of previous $W$ time slices; historical daily and weekly average speed at time $t$ (long term periodicity); time indicator such as time-of-day and day-of-week; holiday indicator (' 1 ' indicates a holiday while ' 0 ' otherwise); and weather conditions in hourly granularity (e.g., raining, sunny, rainstorm, etc.) represented using one-hot coding.

Attributed Decay Network. Similar to $G_{P}^{t}$, the DAN $G_{D}^{t}=\left(V_{D}^{t}, E_{D}^{t}, A D_{D}^{t}, A S_{D}^{t}\right)$ is defined to capture the decay behaviors based on $G_{C}$ at time slots $t-1$, $t$, and $t+1$, as shown in Figure 2 (b). $G_{c}^{t-1}$ contains a congestion cluster $C_{m}^{t}$ consisting of 4 congested road segments that are spatially connected. The congestion cluster contracts over time in the following way. Nodes 2 and 3 released their congestion states from time $t-1$ to $t$, then their neighboring nodes 5 and 6 released their congestion states from time $t$ to $t+1$. In this process, there are two congestion decay paths, i.e., $r_{2} \rightarrow r_{5} \rightarrow r_{6}$ and $r_{3} \rightarrow r_{5} \rightarrow r_{6}$, where nodes 2 and 3 are sources of congestion decay. Formally, a path $p a=\left\langle r_{i_{1}}, . ., r_{i_{k}}, \ldots, r_{i_{K}}\right\rangle$ is a decay path if $\left(i_{j}, i_{j+1}\right) \in E(1 \leq j \leq K-1) ; c_{i_{j}}^{t-1}=1(1 \leq j \leq K)$ $c_{i_{1}}^{t}=0, c_{i_{j}}^{t}=1(2 \leq j \leq K)$, and $c_{i_{j}}^{t}=0(1 \leq j \leq K)$ (consequently released). With the obtained decay paths, $G_{D}^{t}$ can be constructed by including all nodes of the decay paths into set $V_{D}^{t}$ and all links of the decay paths into set $E_{D}^{t} . A D_{D}^{t}$ and $A S_{D}^{t}$ are constructed in the same way as $A D_{P}^{t}$ and $A S_{P}^{t}$.

Congestion Evolution Prediction. The congestion evolution prediction problem is formulated as a link prediction problem on DAN: Given a DAN (e.g., $G_{P}, G_{D}$ ), predict the existence of directed links from a road segment $r_{i}$ to $r_{j}$, based on dynamic topological structures and diverse node attributes.

\section{DMLM Model for Evolution Prediction}

Figure 1 (b) shows the framework of Deep Meta-Learning Model (DMLM) for predicting congestion evolutions, which consists of 5 main steps: 1) Generating asymmetric initial representation by incorporating multiple temporal correlations while preserving structure and asymmetric transitivity; 2) Learning multiple evolving temporal correlations using meta-LSTM modules based on static and dynamic node attributes; 3) Learning time-varying significance for fusing het- erogeneous features using meta-attention modules; 4) Generating final source and target representations by fusing learned latent features as well as their time-varying significance. 5) Predicting the propagation/decay between road pairs based on the obtained representations. The DMLM models for propagation prediction and decay prediction are trained separately. In the rest of the paper, we discuss how to build the model for propagation prediction. The model for decay prediction is obtained in the same way.

\subsection{Asymmetric Initial Representations}

As the congestion propagation is inherently asymmetric, i.e., the possibility of propagation from $r_{i}$ to $r_{j}$ is different from that of $r_{j}$ to $r_{i}$, we propose to learn asymmetric representations [Ou et al., 2016; Zhou et al., 2017] for the road segments. For an APN $G_{P}$, we generate a source representation $\mathbf{e p}_{i, t}^{s r c}$ and a target representation $\mathbf{e p}_{i, t}^{t r g}$ for each node $r_{i}$ at time $t$. Then a function $\phi$ takes $\mathbf{e p}_{i, t}^{s r c}$ and $\mathbf{e p}_{j, t}^{t r g}$ as input variables to predict the likelihood of existence of propagation from $r_{i}$ and $r_{j}$. Due to the asymmetric property, $\phi\left(\mathbf{e p}_{i, t}^{s r c}, \mathbf{e p}_{j, t}^{t r g}\right) \neq \phi\left(\mathbf{e p}_{j, t}^{s r c}, \mathbf{e p}_{i, t}^{t r g}\right) \in[0,1]$.

Three auxiliary matrices are constructed to capture diverse temporal dependencies of evolutions, e.g., recent dependency, daily periodicity, and weekly repeatability [Sun et al., 2019; Lv et al., 2018]. Given APN $G_{P}$, we build 3 matrices for each time slice $t$, i.e., recent, daily and weekly propagation trend matrices: $M_{P, r e}^{t}, M_{P, d a}^{t}$, and $M_{P, w e}^{t}$. Specifically, the matrix $M_{P, r e}^{t}, M_{P, d a}^{t}$ and $M_{P, w e}^{t}$ characterize recent traffic, daily periodic and weekly periodic patterns, respectively. They are constructed as $f(l x, T x)=\frac{1}{l x} \sum_{l=1}^{l x} M_{P}^{t-T x * l}$, where $M_{P}^{t} \in\{0,1\}^{N \times N}$ and $M_{P}^{t}(i, j)=1$ when $\{i, j\} \in$ $E_{P}^{t}$ of $G_{P}, l x$ is the number of previous days/weeks considered, $T x$ is the number of intervals per day/week $(T x=288$ for one day when the time slot is 5 minutes ). For $M_{P, r e}^{t}$, $(l x, T x)=(6,1)$, for $M_{P, d a}^{t},(l x, T x)=(7,288)$, and for $M_{P, w e}^{t},(l x, T x)=(6,288 * 7)$. The aforementioned parameters are based on the settings from [Guo et al., 2019].

$M_{P, r e}^{t}, M_{P, d a}^{t}$ and $M_{P, w e}^{t}$ are fed into an Non-negative Matrix Factorization (NMF) module (as shown in Figure 3 (a) [Lee and Seung, 2001] to generate initial representations (3 source representations and 3 target representations corresponding to $M_{P, r e}^{t}, M_{P, d a}^{t}$ and $M_{P, w e}^{t}$ respectively).

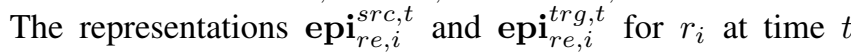
is obtained based on $M_{P, r e}^{t}$ by solving an objective function: $\min \left\|M_{P, r e}^{t}-\mathbf{S}_{r e}^{t}\left(\mathbf{T}_{r e}^{t}\right)^{T}\right\|^{2}$, s.t. $\mathbf{S}_{r e}^{t}, \mathbf{T}_{r e}^{t} \geq 0$, where epi $_{r e, i}^{s r c, t} \in \mathbb{R}^{K}$ is the $i$ th row of matrix $S \in \mathbb{R}^{N \times K}$, and $\mathbf{e p i}_{r e, i}^{t r g, t}$ is the $i$ th row of the transposition of matrix $\mathbf{T} \in$ $\mathbb{R}^{K \times N}$ (i.e., $\left.(\mathbf{T})^{\prime}\right)$. epi $\mathbf{i}_{d a, i}^{s r c, t}, \mathbf{e p i}_{d a, i}^{\operatorname{trg}, t}, \mathbf{e p i} \mathbf{i}_{w e, i}^{s r c, t}$, and $\mathbf{e p i} \mathbf{i}_{w e, i}^{\operatorname{trg}, t}$ are calculated in the same way.

\subsection{Meta-LSTM Component}

The constructed APN $G_{P}$ and ADN $G_{D}$ have included diverse factors (attributes), which can be used to identify impressionable congestion evolutions. This section introduces a Meta-LSTM module to learn the evolving temporal correlations from multiple views. As shown in Figure 3 (b), 


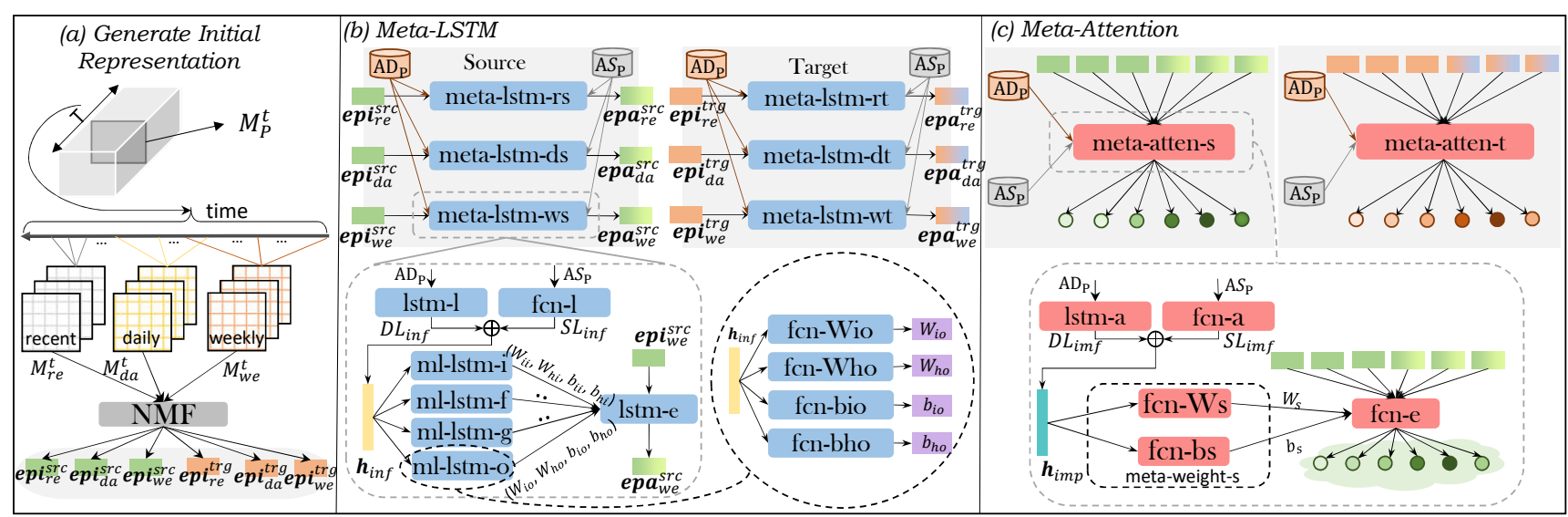

Figure 3: The three main components of DMLM: (a) Generating asymmetric initial representation, (b) Learning evolving temporal correlations using meta-LSTM, (c) Learning time-varying significance for feature fusion using meta-attention.

the Meta-LSTM component processes the source representation part and the target representation part separately, using two separate modules of the same structure. Each part has three separate meta-lstm modules, which fuse multi-view temporal correlation information (recent, daily, and weekly patterns) using heterogeneous weights that are learned via meta-learning based on diverse node attributes of APN $G_{P}$. Taking meta-lstm-ws for learning source representation based on $G_{P}^{t}$ as an example, the inputs involve three parts: representation of weekly propagation trend epi $\mathbf{i}_{w e}^{s r c}$, static attributes $A S_{P}$, and dynamic attributes $A D_{P}$. meta-lstm-rs produces epa $\mathbf{a}_{w e}^{s r c} \in \mathbb{R}^{K}$.

As shown in Figure 3 (b), meta-lstm-we consists of a 3-layers LSTM module $1 \mathrm{stm}-1$ that captures long-term and short-term dependency of dynamic attributes, a 2-layers FCN $\mathrm{f} \mathrm{Cn}-1$ that captures latent impacts of static attributes, and four meta learners for learning parameters in meta-LSTM (i.e., lstm-e). At each time step, the lstm-l learns a latent vector $D L_{\text {inf }} \in \mathbb{R}^{D l_{o}^{m l}}$ from node $r_{i}$ 's dynamic attributes $A D_{P}^{t}(i) \in \mathbb{R}^{F_{d}}$ (the $i$-th row of $A D_{P}^{t}$ ), which incorporates dynamic latent information. The $\mathrm{f} \mathrm{Cn}-1$ learns a $S L_{\text {inf }} \in \mathbb{R}^{D f_{o}^{m l}}$ from $A S_{P}(i) \in \mathbb{R}^{F_{s}}$ to incorporate static latent information. The knowledge learned from both static and dynamic attributes are concatenated before being fed into the four meta-learners, i.e., $\mathbf{h}_{\text {inf }}=D L_{\text {inf }} \oplus S L_{i n f}$. The four meta-learners learn weight parameters for input gate, forget gate, cell, and output gate in lstm-e module, based on $\mathbf{h}_{\text {inf }}$. Based on the learned weights from meta leaners, the lstm-e takes epi $\mathbf{i}_{w e}^{s r c}$ as input to generate $\mathbf{e p a}_{w e}^{s r c}$.

The four meta learners are of the same structure as shown in Figure 3 (b). For example, the meta learners for output gate $(\mathrm{ml}-\mathrm{lstm}-\mathrm{O})$ relies on four separate 2-layers FCN modules to learn 4 parameters for the output gate. Specifically, the four FCN modules (i.e., $f_{\mathrm{cn}}-\mathrm{wio}, \mathrm{f}_{\mathrm{cn}}$-who, $\mathrm{f}_{\mathrm{cn}}$-bio and $\mathrm{fcn}_{\mathrm{c}-\mathrm{bho}}$ ) take $\mathbf{h}_{\text {inf }}$ as input and produce 4 parameters $\mathbf{W}_{\text {io }} \in \mathbf{R}^{K \times K}, \mathbf{W}_{\text {ho }} \in \mathbb{R}^{K \times K}, \mathbf{b}_{i o} \in \mathbb{R}^{K}$, and $\mathbf{b}_{\text {ho }} \in \mathbb{R}^{K}$ for the output gate of lstm-e. In the same way, the parameters of 1 stm-e's other three parts (input gate, forget gate, cell) are learned using 3 meta learners. Then, lstm-e takes epi $\mathbf{i}_{w e}^{s r c}$ as input and produces epa $\mathbf{a}_{w e}^{s r c}$ based on following equations:

$$
\begin{aligned}
\mathbf{i}_{t} & =\sigma\left(\mathbf{W}_{i i} \mathbf{x}_{t}+\mathbf{b}_{i i}+\mathbf{W}_{h i} \mathbf{h}_{t-1}+\mathbf{b}_{h i}\right), \\
\mathbf{f}_{t} & =\sigma\left(\mathbf{W}_{i f} \mathbf{x}_{t}+\mathbf{b}_{i f}+\mathbf{W}_{h f} \mathbf{h}_{t-1}+\mathbf{b}_{h f}\right), \\
\mathbf{g}_{t} & =\sigma\left(\mathbf{W}_{i g} \mathbf{x}_{t}+\mathbf{b}_{i g}+\mathbf{W}_{h g} \mathbf{h}_{t-1}+\mathbf{b}_{h g}\right), \\
\mathbf{o}_{t} & =\sigma\left(\mathbf{W}_{i o} \mathbf{x}_{t}+\mathbf{b}_{i o}+\mathbf{W}_{h o} \mathbf{h}_{t-1}+\mathbf{b}_{h o}\right), \\
\mathbf{C}_{t} & =\mathbf{f}_{t} \circ \mathbf{C}_{t-1}+\mathbf{i}_{t} \circ \mathbf{g}_{t}, \\
\mathbf{h}_{t} & =\mathbf{o}_{t} \circ \tanh \left(\mathbf{C}_{t}\right) .
\end{aligned}
$$

where $\circ$ is Hadamard product, and $\sigma$ is sigmoid functuion.

\subsection{Meta-Attention Component}

APN $G_{P}$ has included various factors that impact the traffic situations. The dynamic as well as the static attributes have time-varying impact on the congestion evolutions (as highlighted earlier). To capture such dynamic significance, we designed a Meta-Attention component to merge multiple temporal correlations as well as static and dynamic attributes. As shown in Figure 3 (c), Meta-Attention component relies on two separate meta-attention modules to learn source and target representations (meta-atten-s and meta-atten-t). In meta-atten-s, a 3-layers LSTM layer (lstm-a) is used to learn a latent vector representation $\left(D L_{i m p} \in \mathbb{R}^{D f_{o}^{m a}}\right)$ for dynamic attributes and a 2-layers FCN layer ( $\mathrm{f} \mathrm{Cn}-\mathrm{a}$ ) is used to learn a latent vector representation $\left(S L_{i m p} \in \mathbb{R}^{D f_{o}^{m a}}\right)$ for the static attributes. Then, $\mathbf{h}_{i m p}=D L_{i m p} \oplus S L_{i m p}$ is fed into a meta learner, meta-weight-s, to learn weight parameters for the attention module $\mathrm{f}_{\mathrm{Cn}}-\mathrm{e}$. The meta learner uses two separate twolayers FCNs, $\mathrm{fcn}_{\mathrm{C}} \mathrm{Ws}$ and $\mathrm{f}_{\mathrm{cn}}-\mathrm{bs}$, to learn weight matrix and bias terms for $\mathrm{f}_{\mathrm{cn}}-\mathrm{e}$. Then, $\mathrm{f}_{\mathrm{cn}}-\mathrm{e}$ takes three initial representations $\left(\mathbf{e p i} \mathbf{i}_{r e}^{s r c}, \mathbf{e p i} \mathbf{i}_{d a}^{s r c}, \mathbf{e p i} \mathbf{i}_{w e}^{s r c}\right)$ together with three

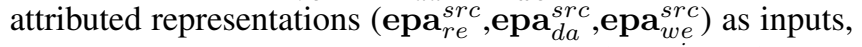
and produces corresponding six scores $a_{r e}^{e p i}, a_{d a}^{e p i}, a_{w e}^{e p i}, a_{r e}^{e p a}$, $a_{d a}^{e p a}$ and $a_{w e}^{e p a}$ based on the following equations:

$a_{x}^{y}=\frac{\exp \alpha_{x}^{y}}{\sum_{l, m} \exp \alpha_{l}^{m}}, x, l \in\{r e, d a, w e\}, y, m \in\{$ epi, epa $\}$.

$\alpha_{x}^{y}=\mathrm{fcn}-\mathrm{e}\left(y_{x}\right), x \in\{r e, d a, w e\}, y \in\{e p i, e p a\}$. 
With the Meta-Attention module, each node in $G_{P}$ obtains six attention scores for source representation learning: $a_{r e, s r c}^{e p i}, a_{d a, s r c}^{e p i}, a_{w e, s r c}^{e p i}, a_{r e, s r c}^{e p a}, a_{d a, s r c}^{e p a}, a_{w e, s r c}^{e p a}$; and six attention scores for target representation learning: $a_{r e, t r g}^{e p i}$, $a_{d a, t r g}^{e p i}, a_{w e, t r g}^{e p i}, a_{r e, t r g}^{e p a}, a_{d a, t r g}^{e p a}, a_{w e, t r g}^{e p a}$.

\subsection{Fusion and Prediction}

For a road segment $r_{i}$ at time $t$ in APN $G_{P}$, a source representation $\mathbf{e p}^{s r c} \in \mathbb{R}^{K}$ and a target representation $\mathbf{e p}^{\text {trg }} \in \mathbb{R}^{K}$ are obtained via the following fusion process.

$$
\begin{aligned}
& \mathbf{e p}^{s r c}=\sum_{x, y} a_{x, s r c}^{y} * y_{x}^{s r c}, x \in\{r e, d a, w e\}, y \in\{\mathbf{e p i}, \mathbf{e p a}\} . \\
& \mathbf{e p}^{\operatorname{trg}}=\sum_{x, y} a_{x, t r g}^{y} * y_{x}^{t r g}, x \in\{r e, d a, w e\}, y \in\{\mathbf{e p i}, \mathbf{e p a}\} .
\end{aligned}
$$

The likelihood of a directed link from $r_{i}$ to $r_{j}$ is calculated as inner product of $r_{i}$ 's source representation and $r_{j}$ 's target representation, i.e., $y^{p}(i, j)=\sigma\left(\mathbf{e p}_{i}^{s r c} \cdot \mathbf{e p}_{j}^{t r g}\right), \sigma$ is sigmoid function. There exists a link if $y^{p}(i, j) \geq 0.5$ and no link otherwise.

Loss function . The loss function for training DMLM to predict propagation behavior is defined as follows.

$$
L_{P}=-\frac{1}{n} \sum_{k=1}^{n}{\hat{y^{p}}}_{k} \cdot \ln y_{k}^{p}+\left(1-\hat{y}_{k}^{p}\right) \cdot \ln \left(1-y_{k}^{p}\right)
$$

where $n$ is the number of samples, $y^{p}{ }_{k}$ is prediction, and $\hat{y}^{p}{ }_{k}$ is ground truth.

\section{Experiments}

\subsection{Dataset}

Road Network. The road network for our experiments is obtained from OpenStreetMap ${ }^{1}$, which covers a rectangle area in Downtown of Singapore (Southwest: 1.2718, 103.8002; Northeast: $1.3323,103.8653$ ) consisting of 1858 road segments. The topological information and static attributes of roads are extracted (as discussed in Section 3).

POIs. The POIs are collected from government website ${ }^{2}$. We group all POIs into 14 categories: shopping services, religion building, business, hotel, residence, education, food, government, scenic spot, medical care, sports, entertainment, bus stop, MRT station.

Traffic Data. The historical traffic speeds are calculated based on bus trajectories derived from bus arrival data ${ }^{3}$. Speeds are aggregated every 5 minutes. Then, a road segment is detected as congested at a time interval if its traffic speed is slower than a threshold value. Similar to existing works[Nguyen et al., 2016], we tested several thresholds, i.e., for $r_{i}$, threshold $\theta_{i}^{p}$ include different percentiles, denoted as p. $p=90 \%$ indicates that $90 \%$ of the $r_{i}$ 's speed values in $\mathbf{s}_{i}$

\footnotetext{
${ }^{1} \mathrm{https}: / /$ www.openstreetmap.org/export

${ }^{2} \mathrm{https}: / /$ data.gov.sg/dataset?q=Places+of+Interest

${ }^{3}$ https://www.mytransport.sg/content/mytransport/home/dataMall .html
}
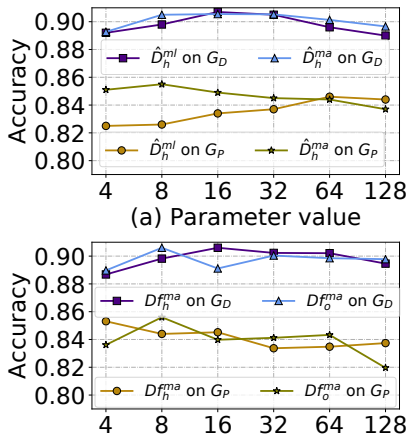

(c) Parameter value

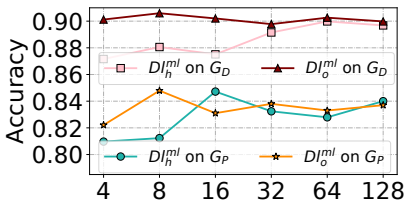

(b) Parameter value

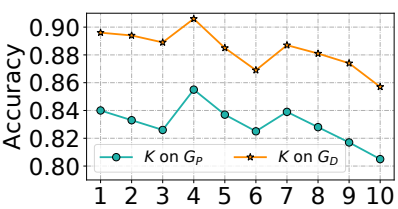

(d) Parameter value
Figure 4: Impacts of model parameters.

are larger than $\theta_{i}^{p}$. Thus, $10 \%$ of $r_{i}$ 's speeds will be identified as congested in $\mathbf{c}_{i}$. The traffic data is collected from Aug. 01 to Nov. 30, 2018, where the data of the first 90 days (75\%) is used for training and the remaining data is used for testing.

\subsection{Evaluation Metrics and Baseline Methods}

Metrics. The performance of the proposed methods is evaluated using metrics accuracy and F1-score.

Baselines. 1) STC [Nguyen et al., 2016] extracts frequently appearing congestion propagation relationship by mining frequent subtrees. Then, a dynamic Bayesian network is constructed using the obtained patterns to predict propagations. 2) Pro-Graph [Xiong et al., 2018] works on a graph structure and predicts where certain congestions will propagate to the near future. 3) MMDNE [Lu et al., 2019] proposes temporal embedding method to incorporate evolution of dynamic networks at micro- and macro-level, and predicts links between node pairs. 4) GCN-GAN [Lei et al., 2019] considers non-linear characteristics and link weights in dynamic network leveraging on graph convolutional network (GCN), LSTM and generative adversarial network (GAN) for link prediction. 5) ANRL [Zhang et al., 2018] employs a neighbor enhancement autoencoder to model node attributes and a skip-gram model to formulate structures for learning node representations. Link prediction is then performed based on cosine similarity function. 6) node2bits [Jin et al., 2019] uses a time- and attribute-aware framework to study interactions of users in heterogeneous networks and predict whether two users correspond to the same entity. The first two baselines are the most recent works that investigate congestion evolution prediction problems. Baselines 3) and 4) are link prediction methods considering temporal dimension properties. Baseline 5) incorporates node attributes but ignore properties over temporal dimension, while baseline 6) considers both node attributes and temporal attributes. We also include the ablation version of our method by removing major components. 7) Ours-woML (without meta-LSTM) employs the original LSTM instead of the meta-LSTM structure. 8) OurswoMA (without meta-Attention) employs a widely used attention mechanism instead of the proposed meta-Attention structure. 


\begin{tabular}{l|rrr|rrr}
\hline & \multicolumn{3}{|c|}{ Accuracy } & \multicolumn{3}{c}{ F1-score } \\
\cline { 2 - 7 } & $90 \%$ & $75 \%$ & $60 \%$ & $90 \%$ & $75 \%$ & $60 \%$ \\
\hline STC & 0.652 & 0.648 & 0.671 & 0.685 & 0.677 & 0.769 \\
\hline Pro-Graph & $\mathbf{0 . 8 6 0}$ & 0.692 & 0.547 & 0.097 & 0.117 & 0.118 \\
\hline MMDNE & 0.487 & 0.504 & 0.496 & 0.396 & 0.403 & 0.469 \\
\hline GCN-GAN & 0.503 & 0.501 & 0.508 & 0.014 & 0.004 & 0.034 \\
\hline ANRL & 0.499 & 0.50 & 0.498 & 0.665 & 0.667 & 0.663 \\
\hline Node2Bits & 0.518 & 0.542 & 0.534 & 0.635 & 0.675 & 0.667 \\
\hline \hline Ours-woML & 0.830 & 0.804 & 0.837 & 0.855 & 0.829 & 0.850 \\
\hline Ours-wOMA & 0.851 & 0.849 & 0.863 & 0.863 & 0.857 & 0.871 \\
\hline Ours & 0.859 & $\mathbf{0 . 8 6 2}$ & $\mathbf{0 . 8 7 6}$ & $\mathbf{0 . 8 7 2}$ & $\mathbf{0 . 8 7 4}$ & $\mathbf{0 . 8 8 3}$ \\
\hline
\end{tabular}

Table 1: Overall performance on congestion propagation prediction.

\begin{tabular}{l|rrr|rrr}
\hline & \multicolumn{3}{|c|}{ Accuracy } & \multicolumn{3}{c}{ F1-score } \\
\cline { 2 - 7 } & $90 \%$ & $75 \%$ & $60 \%$ & $90 \%$ & $75 \%$ & $60 \%$ \\
\hline MMDNE & 0.375 & 0.434 & 0.397 & 0.319 & 0.314 & 0.318 \\
\hline GCN-GAN & 0.501 & 0.503 & 0.501 & 0.003 & 0.003 & 0.003 \\
\hline ANRL & 0.497 & 0.50 & 0.499 & 0.661 & 0.665 & 0.662 \\
\hline Node2Bits & 0.489 & 0.487 & 0.487 & 0.154 & 0.305 & 0.274 \\
\hline \hline Ours-woML & 0.798 & 0.752 & 0.698 & 0.843 & 0.704 & 0.531 \\
\hline Ours-woMA & 0.883 & 0.864 & 0.852 & 0.892 & 0.877 & 0.870 \\
\hline Ours & $\mathbf{0 . 9 3 9}$ & $\mathbf{0 . 9 0 6}$ & $\mathbf{0 . 8 8 7}$ & $\mathbf{0 . 9 4 2}$ & $\mathbf{0 . 9 1 3}$ & $\mathbf{0 . 8 9 7}$ \\
\hline
\end{tabular}

Table 2: Overall performance on congestion decay prediction.

\subsection{Experiment Setup and Hyper-parameters}

We implemented the DMLM model using PyTorch framework on Intel(R) Xeon(R) CPU E5-1650 v2 @ $3.50 \mathrm{GHz}$ with $32 \mathrm{G}$ RAM. The source code is available at https://github.com/HelenaYD/DMLM. The critical hyperparameters are optimized via grid search as follows. Size of hidden layers of meta learners's FCNs in Meta-LSTM and Meta-Attention: $\hat{D}_{h}^{m l}=64, \hat{D}_{h}^{m a}=8$; Size of hidden and output layers in 1 stm-l and lstm-a: $D l_{h}^{m l}=16, D l_{o}^{m l}=8, D l_{h}^{m a}=8, D l_{o}^{m a}=32$; Size of hidden and output layers in $\mathrm{f}_{\mathrm{Cn}}-1$ and $\mathrm{f}_{\mathrm{Cn}}-\mathrm{a}: D f_{h}^{m l}=4$, $D f_{o}^{m l}=16, D f_{h}^{m a}=4, D f_{o}^{m a}=8$; and the dimension of final representations $K=4$. For decay prediction, the above parameters are set to: $16,16,64,8,128,8,64,16,16,8$, respectively. In addition, the learning rate is 0.0001 and the batch size is 40 .

\subsection{Results and Analysis}

Table 1 and Table 2 show the comparison with 6 baselines and 2 ablation versions of our method. In addition to testing the prediction performance on congestion propagation, we also predict the congestion decay by training another DMLM using $\mathrm{ADN} G_{D}$. The results show that our method outperforms all the baselines in both accuracy or f1-score for both propagation and decay prediction. STC and Pro-Graph are designed for congestion propagation, and cannot be easily extended to decay prediction. Thus we only tested their performance on propagation prediction. Pro-Graph obtains comparable accuracy with our DMLM when $p=90 \%$ as it relies on propagation patterns (e.g., repeatability) to make predictions. As such, it produces higher accuracy for heavy congestions that repeat frequently, and the accuracy decreases rapidly with decreasing $p$. Both MMDNE and GCN-GAN considered temporal dependency and structure information but failed to obtain satisfying results. This indicates that it is critical to incorporate diverse node attributes in addition to the temporal dependency and network structure information. On the other hand, ANRL considered node attributes but ignore temporal dimension, leading to poor performance. In general, the baselines produce low-quality solutions because they either did not sufficiently incorporate the spatiotemporal correlations or failed to capture the dynamic significances of both the dynamic and static node attributes. On the other hand, Ours-woML and Ours-woMA produce obvious performance degradation for both propagation and decay prediction. This demonstrates that the Meta-LSTM and Meta-Attention modules play important roles in the DMLM. In addition, combining NMF with diverse temporal dependencies captures the underlying evolution trends, which makes prediction more stable.

Figure 4 illustrates the impacts of several factors on the prediction performance, including $\hat{D}_{h}^{m l}, \hat{D}_{h}^{m a}, D l_{h}^{m l}, D l_{o}^{m l}$, $D f_{h}^{m a}, D f_{o}^{m a}$, and $K$. These factors affect the dimensions of intermediate feature vectors. As shown in Figure 4 (a), increasing $\hat{D}_{h}^{m l}$ and $\hat{D}_{h}^{m a}$ first leads to perform improvement, and then results in performance degradation. In general, feature vectors that are too short cannot sufficiently characterize the complex correlations, while vectors that are too long not only fail to improve representation capability, but also lead to higher computation overhead. The parameters, e.g., $D l_{h}^{m l}$ and $D l_{o}^{m l}$ in Figure 4 (c), $D f_{h}^{m a}$ and $D f_{o}^{m a}$ in (d), also produce similar trends (the other parameters are not shown due to the page limit). The figure also shows that even under similar trends, the best parameter setting for propagation prediction is different from decay prediction.

\section{Conclusion}

This paper proposed methods to model and predict the likelihood of congestion evolution between any pair of road segments (connected via single or multiple paths). Dynamic attributed networks were constructed to incorporate both dynamic and static impact factors while preserving dynamic topological structures. A DMLM model was proposed to learn representations of road segments, which enable accurate prediction of congestion evolution. DMLM relies on meta-LSTM and meta-Attention techniques to incorporate the heterogeneous and time-varying impacts of multiple correlations, and various dynamic and static node attributes. Experiments on real-world datasets showed that the proposed method achieves significantly better results than all state-ofthe-art methods.

\section{Acknowledgements}

This research project is supported in part by the National Research Foundation Singapore under its Campus for Research Excellence and Technological Enterprise (CREATE) programme with the Technical University of Munich at TUMCREATE.

\section{References}

[An et al., 2016] Shi An, Haiqiang Yang, Jian Wang, Na Cui, and Jianxun Cui. Mining urban recurrent congestion evo- 
lution patterns from gps-equipped vehicle mobility data. Inform. Sciences, 373:515-526, 2016.

[An et al., 2018] Shi An, Haiqiang Yang, and Jian Wang. Revealing recurrent urban congestion evolution patterns with taxi trajectories. ISPRS Interl. J. of Geo-Inform., 7(4):128, 2018.

[Anwar et al., 2016] Tarique Anwar, Chengfei Liu, Hai L $\mathrm{Vu}$, and Md Saiful Islam. Tracking the evolution of congestion in dynamic urban road networks. In CIKM, pages 2323-2328, 2016.

[Cui et al., 2019] Zhiyong Cui, Kristian Henrickson, Ruimin Ke, and Yinhai Wang. Traffic graph convolutional recurrent neural network: A deep learning framework for network-scale traffic learning and forecasting. IEEE Transactions on Intelligent Transportation Systems, 21(11):4883-4894, 2019.

[Di et al., 2019] Xiaolei Di, Yu Xiao, Chao Zhu, Yang Deng, Qinpei Zhao, and Weixiong Rao. Traffic congestion prediction by spatiotemporal propagation patterns. In $M D M$, pages 298-303. IEEE, 2019.

[Guo et al., 2019] Shengnan Guo, Youfang Lin, Ning Feng, Chao Song, and Huaiyu Wan. Attention based spatialtemporal graph convolutional networks for traffic flow forecasting. In $A A A I$, volume 33, pages 922-929, 2019.

[Jin et al., 2019] Di Jin, Mark Heimann, Ryan A Rossi, and Danai Koutra. node2bits: Compact time-and attributeaware node representations for user stitching. In ECMLPKDD, pages 483-506. Springer, 2019.

[Khajeh-Hosseini and Talebpour, 2019] Mohammadreza Khajeh-Hosseini and Alireza Talebpour. Back to the future: Predicting traffic shockwave formation and propagation using a convolutional encoder-decoder network. In ITSC, pages 1367-1372. IEEE, 2019.

[Lee and Seung, 2001] Daniel D Lee and H Sebastian Seung. Algorithms for non-negative matrix factorization. In NeurIPS, pages 556-562, 2001.

[Lei et al., 2019] Kai Lei, Meng Qin, Bo Bai, Gong Zhang, and Min Yang. Gen-gan: A non-linear temporal link prediction model for weighted dynamic networks. In INFOCOM, pages 388-396. IEEE, 2019.

[Li et al., 2018] Jundong Li, Kewei Cheng, Liang Wu, and Huan Liu. Streaming link prediction on dynamic attributed networks. In WSDM, pages 369-377, 2018.

[Liao et al., 2018] Binbing Liao, Jingqing Zhang, Chao Wu, Douglas McIlwraith, Tong Chen, Shengwen Yang, Yike Guo, and Fei Wu. Deep sequence learning with auxiliary information for traffic prediction. In KDD, pages 537-546, 2018.

[Lichtenwalter et al., 2010] Ryan N Lichtenwalter, Jake T Lussier, and Nitesh V Chawla. New perspectives and methods in link prediction. In KDD, pages 243-252, 2010.

[Lu et al., 2019] Yuanfu Lu, Xiao Wang, Chuan Shi, Philip S $\mathrm{Yu}$, and Yanfang Ye. Temporal network embedding with micro-and macro-dynamics. In CIKM, pages 469-478, 2019.
[Lv et al., 2018] Zhongjian Lv, Jiajie Xu, Kai Zheng, Hongzhi Yin, Pengpeng Zhao, and Xiaofang Zhou. Lcrnn: A deep learning model for traffic speed prediction. In IJCAI, pages 3470-3476, 2018.

[Martínez et al., 2016a] Víctor Martínez, Fernando Berzal, and Juan-Carlos Cubero. Adaptive degree penalization for link prediction. Journal of Computational Science, 13:19, 2016.

[Martínez et al., 2016b] Víctor Martínez, Fernando Berzal, and Juan-Carlos Cubero. A survey of link prediction in complex networks. ACM computing surveys (CSUR), 49(4):1-33, 2016.

[Nguyen et al., 2016] Hoang Nguyen, Wei Liu, and Fang Chen. Discovering congestion propagation patterns in spatio-temporal traffic data. IEEE Trans. Big Data, 3(2):169-180, 2016.

[Ou et al., 2016] Mingdong Ou, Peng Cui, Jian Pei, Ziwei Zhang, and Wenwu Zhu. Asymmetric transitivity preserving graph embedding. In $K D D$, pages 1105-1114, 2016.

[Sun et al., 2018] Jian Sun, Zian Ma, and Xuemei Chen. Some observed features of traffic flow phase transition at urban expressway diverge bottlenecks. TRANSPORTMETRICA B, 6(4):320-331, 2018.

[Sun et al., 2019] Yidan Sun, Guiyuan Jiang, Siew-Kei Lam, Shicheng Chen, and Peilan He. Bus travel speed prediction using attention network of heterogeneous correlation features. In Proceedings of the 2019 SIAM International Conference on Data Mining, pages 73-81. SIAM, 2019.

[Xiong et al., 2018] Haoyi Xiong, Amin Vahedian, Xun Zhou, Yanhua Li, and Jun Luo. Predicting traffic congestion propagation patterns: A propagation graph approach. In SIGSPATIAL, pages 60-69, 2018.

[Yue et al., 2018] Wenwei Yue, Changle Li, and Guoqiang Mao. Urban traffic bottleneck identification based on congestion propagation. In ICC, pages 1-6. IEEE, 2018.

[Zhang et al., 2017] Tianqi Zhang, Lishan Sun, Liya Yao, and Jian Rong. Impact analysis of land use on traffic congestion using real-time traffic and poi. Journal of $\mathrm{Ad}$ vanced Transportation, 2017, 2017.

[Zhang et al., 2018] Zhen Zhang, Hongxia Yang, Jiajun Bu, Sheng Zhou, Pinggang Yu, Jianwei Zhang, Martin Ester, and Can Wang. Anrl: Attributed network representation learning via deep neural networks. In IJCAI, volume 18, pages 3155-3161, 2018.

[Zhou et al., 2017] Chang Zhou, Yuqiong Liu, Xiaofei Liu, Zhongyi Liu, and Jun Gao. Scalable graph embedding for asymmetric proximity. In AAAI, pages 2942-2948, 2017. 